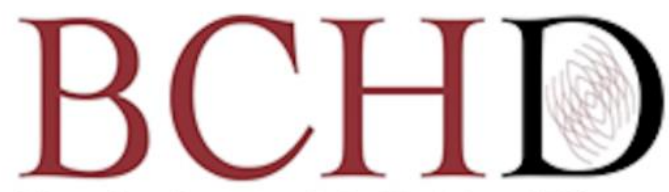

Bioactive Compounds in Health and Disease

\title{
Comparison of the anti-inflammatory activities of furanocoumarins from the roots of Angelica dahurica
}

\section{Ryo Okada ${ }^{1}$, Hazuki Abe ${ }^{1}$, Tetsuya Okuyama ${ }^{1,2}$, Yuto Nishidono ${ }^{3}$, Toshinari Ishii ${ }^{1}$, Tatsuki Sato ${ }^{1}$, Saki Shirako ${ }^{1}$, Ken Tanaka ${ }^{3}$, Yukinobu Ikeya ${ }^{4}$, Mikio Nishizawa ${ }^{1 *}$}

\begin{abstract}
${ }^{1}$ Department of Biomedical Sciences, College of Life Sciences, Ritsumeikan University, Kusatsu, Shiga 525-8577, Japan; ${ }^{2}$ Department of Surgery, Kansai Medical University, Hirakata, Osaka 573-1010, Japan; ${ }^{3}$ College of Pharmaceutical Sciences, Ritsumeikan University, Kusatsu, Shiga 525-8577, Japan; ${ }^{4}$ Center for Supporting Pharmaceutical Education, Daiichi University of Pharmacy, Minami-ku, Fukuoka 815-8511, Japan
\end{abstract}

${ }^{*}$ Corresponding Author: Professor Mikio Nishizawa, M.D., Ph.D., Department of Biomedical Sciences, College of Life Sciences, Ritsumeikan University; Kusatsu, Shiga 525-8577, Japan

Submission Date: November 15 ${ }^{\text {th }}, 2021$; Acceptance Date: December 15 th, 2021 ; Publication Date: December $20^{\text {th }}, 2021$

Please cite this article as: Okada R., Abe H., Okuyama T., Nishidono Y., Ishii T., Sato T., Shirako S., Tanaka K., Ikeya Y., Nishizawa M. Comparison of the anti-inflammatory activities of furanocoumarins from the roots of Angelica dahurica. Bioactive Compounds in Health and Disease 2021; 4(12): 287-300. DOI: https://www.doi.org/10.31989/bchd.v4i12.866

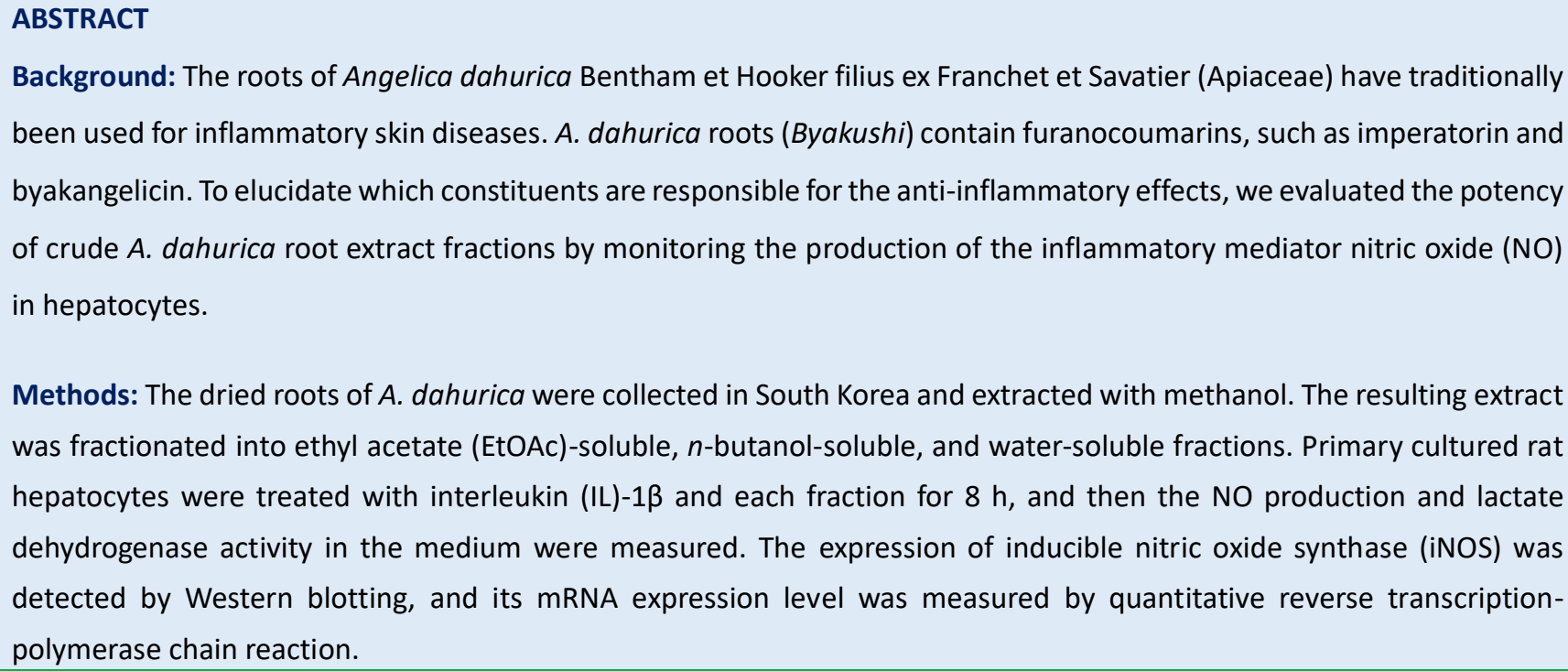
been used for inflammatory skin diseases. A. dahurica roots (Byakushi) contain furanocoumarins, such as imperatorin and byakangelicin. To elucidate which constituents are responsible for the anti-inflammatory effects, we evaluated the potency of crude $A$. dahurica root extract fractions by monitoring the production of the inflammatory mediator nitric oxide (NO) in hepatocytes.

Methods: The dried roots of $A$. dahurica were collected in South Korea and extracted with methanol. The resulting extract was fractionated into ethyl acetate (EtOAc)-soluble, $n$-butanol-soluble, and water-soluble fractions. Primary cultured rat hepatocytes were treated with interleukin (IL)-1 $\beta$ and each fraction for $8 \mathrm{~h}$, and then the NO production and lactate dehydrogenase activity in the medium were measured. The expression of inducible nitric oxide synthase (iNOS) was detected by Western blotting, and its mRNA expression level was measured by quantitative reverse transcriptionpolymerase chain reaction. 
Results: Among the three fractions, the EtOAc-soluble fraction markedly suppressed NO production without showing cytotoxicity and decreased iNOS expression in hepatocytes. From this hydrophobic fraction, we isolated five furanocoumarins: isoimperatorin, imperatorin, phellopterin, oxypeucedanin, and oxypeucedanin methanolate. Phellopterin and oxypeucedanin methanolate significantly suppressed NO production and reduced the mRNA expression of iNOS and tumor necrosis factor $\alpha$. In contrast, the other three constituents did not affect NO production. Comparison of their chemical structures suggests that a methoxy group at carbon 5 and a side chain at carbon 8 in the furanocoumarin skeleton may be essential for NO production suppression.

Conclusion: These data imply that phellopterin and oxypeucedanin methanolate, which are hydrophobic furanocoumarins, may contribute to the anti-inflammatory effects of $A$. dahurica roots by suppressing iNOS gene expression.

Keywords: Inflammation, nitric oxide, hepatocyte, coumarin, Kampo medicine

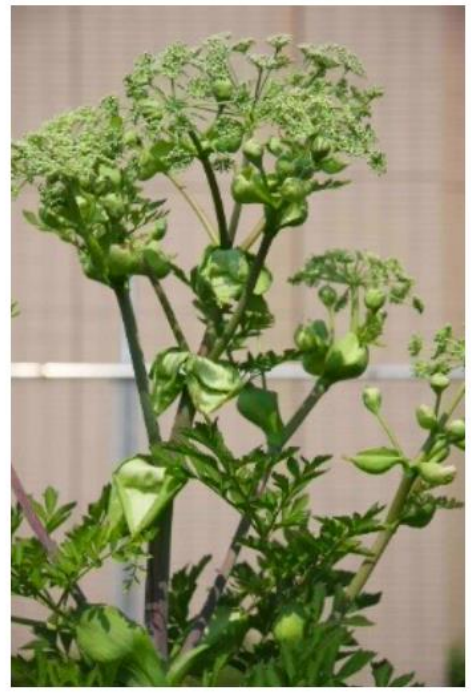

Angelica dahurica (Byakushi)

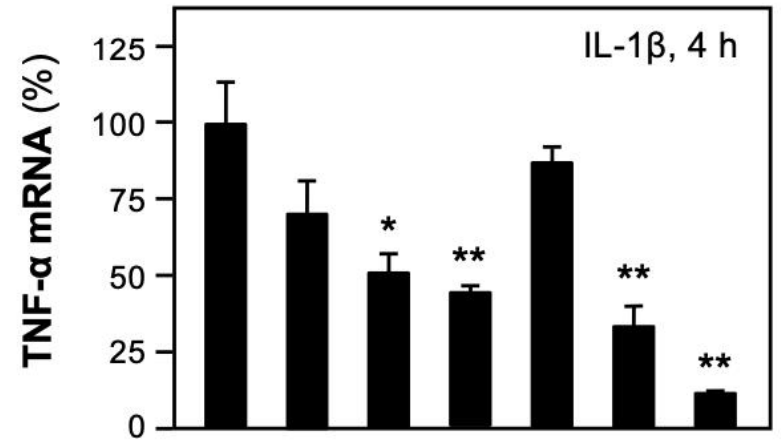

Conc. $(\mu \mathrm{M}) \quad 0 \quad 20 \quad 40 \quad 80 \quad 40 \quad 80 \quad 160$

Compound (-) Phellopterin Oxypeucedanin methanolate

\section{Furocoumarins in the root of Angelica dahurica decrease TNF- $\alpha$ mRNA.}

CFFC 2021. This is an Open Access article distributed under the terms of the Creative Commons Attribution 4.0 License (http://creativecommons.org/licenses/by/4.0)

\section{INTRODUCTION}

Angelica dahurica Bentham et Hooker filius ex Franchet et Savatier (Apiaceae) grows in Japan (known as Yoroigusa), China, and South Korea (Figure 1). Its roots (called Byakushi in Japan) have a bitter taste and are considered to resolve the exterior to emanate pathogens (Gehyou) in traditional Japanese medicine [1]. The roots of $A$. dahurica contain furanocoumarins (also called furocoumarins, e.g., byakangelicin and imperatorin) and monoterpenoids (e.g., $\alpha$-pinene and camphene). The roots of $A$. dahurica have been included in Kampo formulas, such as Seijobofuto, which is used to treat inflammatory skin diseases, including eczema and acne. 
A

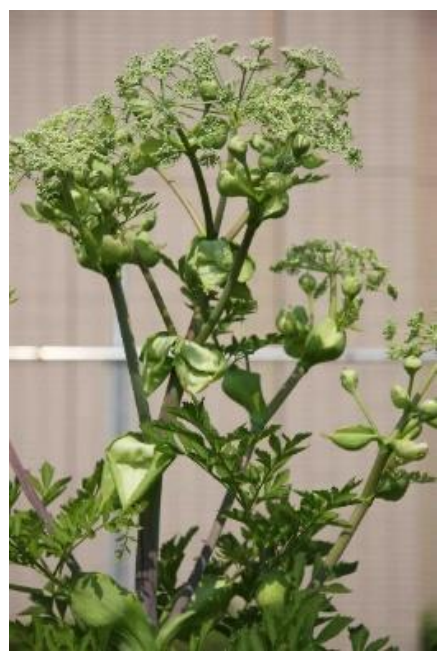

B

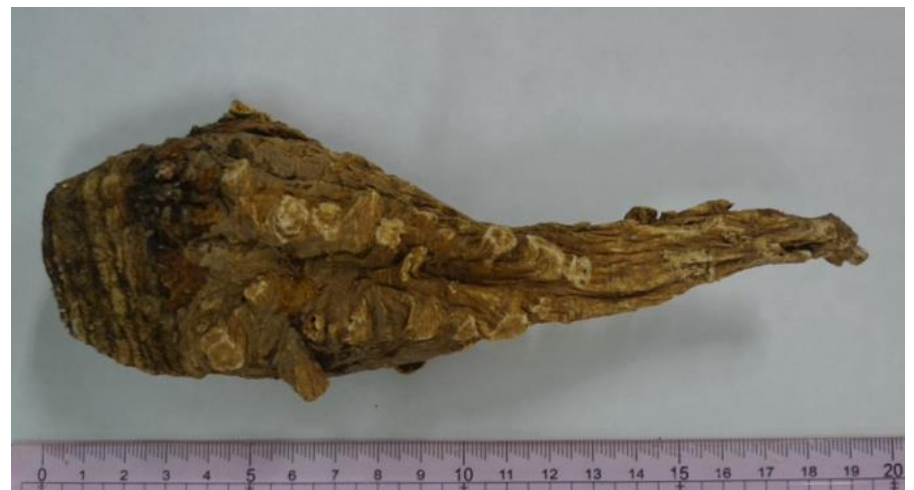

Figure 1. Angelica dahurica Bentham et Hooker filius ex Franchet et Savatier (Apiaceae). (A) An aboveground part of $A$. dahurica, which was grown at Biwako-Kusatsu Campus, Ritsumeikan University, Kusatsu, Shiga, Japan. (B) A root of A. dahurica, which was collected in South Korea. The same lot of $A$. dahurica root was used in this study. Scale is in $\mathrm{cm}$.

Foreign pathogenic bacteria and viruses provoke inflammatory responses. Lipopolysaccharide (LPS) is released from the outer membrane of dead gram-negative bacteria and induces the production of nitric oxide (NO), which is an important proinflammatory mediator synthesized by inducible nitric oxide synthase (iNOS) [2]. In the liver, LPS stimulates Kupffer cells (resident macrophages) to secrete interleukin (IL)- $1 \beta$, which then stimulates hepatocytes to induce the iNOS protein [3]. Primary cultured rat hepatocytes treated with IL-1 $\beta$ are used as an ex vivo system to mimic liver injury. Because the suppression of NO production is correlated with an antiinflammatory effect, $\mathrm{NO}$ is a sensitive marker to estimate the anti-inflammatory activity of constituents in crude drugs and functional foods [4].

It was reported that an ethyl acetate extract from the root of $A$. dahurica inhibited the production of NO and prostaglandin $\mathrm{E}_{2}\left(\mathrm{PGE}_{2}\right)$ in two macrophage lines (human THP-1 and mouse RAW264.7) in the presence of LPS [5]. However, there are few reports comparing the antiinflammatory activities of the constituents of $A$. dahurica roots.

Here, the anti-inflammatory constituents of $A$. dahurica roots were examined, and their effect on NO production in rat hepatocytes was evaluated in the presence of IL-1 $\beta$. The constituents were isolated from $A$. dahurica roots, and we investigated their potency to suppress NO production and the expression of inflammatory genes to clarify the anti-inflammatory effects of $A$. dahurica roots.

\section{MATERIALS AND METHODS}

General experimental procedures: Nuclear magnetic resonance (NMR) spectra were recorded on a JNM-ECS400 NMR spectrometer (JEOL Ltd., Akishima, Tokyo, Japan) operated at $400 \mathrm{MHz}\left({ }^{1} \mathrm{H}\right)$ and $100 \mathrm{MHz}\left({ }^{13} \mathrm{C}\right)$ with tetramethylsilane (TMS) as an internal standard. TMS and deuterated chloroform $\left(\mathrm{CDCl}_{3}\right)$ were purchased from Euriso-Top (Saint-Aubin, France). High-performance liquid chromatography (HPLC) analyses were performed using a Prominence HPLC system with an LC 20AT SPD A UV/VIS detector for preparative HPLC (Shimadzu Corporation, Kyoto, Japan). Semipreparative HPLC was performed using a Shimadzu Prominence HPLC system with an SPD-20A Prominence UV/Vis detector (Shimadzu Corporation) and a Cosmosil Cholester Packed Column [10 mm internal diameter (i.d.) × 250 mm, 5 4m; Nacalai Tesque, Inc., Kyoto, Japan] at a detection wavelength of $254 \mathrm{~nm}$. Column chromatography was run on silica gel 60 (Nacalai Tesque Inc., Kyoto, Japan). Precoated thin-layer chromatography 
(TLC) was performed on silica gel $60 \mathrm{~F}_{254}$ plates (FUJIFILM Wako Pure Chemical Corporation).

Plant material: The roots of $A$. dahurica Bentham et Hooker filius ex Franchet et Savatier (Apiaceae) collected in South Korea were obtained from Tochimoto Tenkaido Co., Ltd. (Osaka, Japan) and authenticated by Dr. Yutaka Yamamoto (Tochimoto Tenkaido Co., Ltd.). A voucher sample was deposited in the Ritsumeikan Herbarium of Pharmacognosy, Ritsumeikan University, under code number RIN-AD-31.

Extraction of the crude drug and fractionation: Dried $A$. dahurica roots (819.3 g) were crushed, pulverized, and extracted with methanol $(\mathrm{MeOH})$ under reflux. The resultant extract was evaporated in vacuo to obtain 282.2 g (34.4\% yield from the dried roots). Briefly, the extract was fractionated based on hydrophobicity, according to a previously established method [6]. The resultant extract was suspended in water and successively extracted with ethyl acetate (EtOAc) and $n$-butanol (Figure 2). Insoluble precipitates were removed from the final aqueous layer. Each layer was collected and concentrated by solvent evaporation to prepare an EtOAc-soluble fraction (fraction A), an $n$-butanol-soluble fraction (fraction B), and a watersoluble fraction (fraction $\mathrm{C}$ ).

\section{Isolation of the constituents from the $\mathrm{MeOH}$ extract:} Fraction A (27.6 g), which showed high activity to suppress NO production in hepatocytes, was further purified by silica gel 60 (70-230 mesh; Nacalai Tesque, Inc.) column chromatography [ $4.0 \mathrm{~cm}$ i.d. $\times 22.5 \mathrm{~cm}$ ] by elution with $n$ hexane:EtOAc $(100: 0 \rightarrow 0: 100)$ to yield 12 subfractions (A1 to $\mathrm{A} 12)$.

Subfractions A2, A5, A7, and A9 were further purified. Subfraction $\mathrm{A} 2$ was recrystallized from $\mathrm{MeOH}$ to give white needles (152 mg), designated as compound 1. Subfraction A7 was recrystallized from $\mathrm{MeOH}$ to give white dendrite crystals (72 mg), designated as compound 2 . Subfraction A5 was subjected to preparative HPLC using a

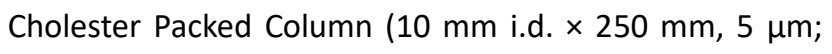
Nacalai Tesque Inc.; mobile phase, acetonitrile:water (60:40); flow rate, $5.0 \mathrm{~mL} / \mathrm{min})$, which resulted in four subfractions, two of which gave compound $\mathbf{3}$ as white needle crystals [retention time $\left(t_{R}\right)=4.9 \mathrm{~min} ; 20 \mathrm{mg}$ ] and compound 4 as yellow dendrite crystals $\left(t_{R}=6.1 \mathrm{~min} ; 15\right.$ $\mathrm{mg}$ ). Subfraction A9 was subjected to silica gel 60 column chromatography by elution with $n$ hexane:EtOAc:chloroform (9:9:2) and then with $n$ hexane:EtOAc:chloroform (3:6:1). Next, preparative TLC was performed with $n$-hexane:EtOAc:chloroform (1:8:1) to obtain compound 5 as yellow dendrite crystals $(20 \mathrm{mg}$ ). The ${ }^{1} \mathrm{H}-\mathrm{NMR}$ and ${ }^{13} \mathrm{C}-\mathrm{NMR}$ spectra were analyzed to identify the compounds by determining their chemical structures.

HPLC analysis of the constituents: The extract and isolated compounds were dissolved in $\mathrm{MeOH}$ at a concentration of $1.0 \mathrm{mg} / \mathrm{ml}$. The solutions were filtered through a $0.45 \mu \mathrm{m}$ Millipore filter unit (Advantec, Tokyo, Japan), and the filtrates $(10 \mu \mathrm{L})$ were injected onto the Cosmosil $5 \mathrm{C}_{18} \mathrm{MS}$ -

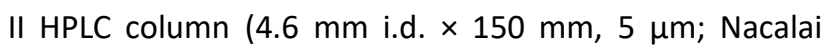
Tesque, Inc.). The UV detection wavelength was $270 \mathrm{~nm}$. The mobile phase was a binary eluent of $(A)$ water and $(B)$ acetonitrile under the following gradient conditions: 0-10 min, linear gradient from $30 \%$ to $45 \%$ B; $10-25$ min, linear gradient from $45 \%$ to $60 \% \mathrm{~B} ; 25-35 \mathrm{~min}$, linear gradient from $60 \%$ to $65 \%$ B; $35-45 \mathrm{~min}$, linear gradient from $65 \%$ to $100 \% \mathrm{~B} ; 45-60 \mathrm{~min}$, isocratic at $100 \% \mathrm{~B}$. The flow rate was $1.0 \mathrm{~mL} / \mathrm{min}$, and the column temperature was $40^{\circ} \mathrm{C}$.

Each compound confirmed by NMR spectral analysis was accurately weighed and dissolved in $\mathrm{MeOH}$ to make $1.00 \mathrm{mg} / 5 \mathrm{~mL}$ stock solutions, and 2.5, 5.0 and $10.0 \mu \mathrm{L}$ of each standard solution were analyzed in triplicate. The calibration curve for each standard compound was constructed by plotting the peak areas $(y)$ against the amount injected $(x)$, and the regression equation was calculated in the form $y=A x+B$ : compound 1 (isoimperatorin), $y=31067674.29 x+13333.50\left(R^{2}=\right.$ 1.0000); 2 (oxypeucedanin), $y=24903707.48 x-533.62\left(R^{2}\right.$ 
$=0.9999) ; 3$ (imperatorin), $y=23508476.19 x+8,781.67\left(R^{2}\right.$

$=1.0000) ; 4$ (phellopterin), $y=41433464.76 x+8451.17\left(R^{2}\right.$

= 1.0000); and 5 (oxypeucedanin methanolate), $y=$ 15407659.86x -521.59 $\left(R^{2}=0.9999\right)$. Fraction $\mathrm{A}$ was accurately weighed and dissolved in $\mathrm{MeOH}$ to prepare a $1.0 \mathrm{mg} / \mathrm{mL}$ solution and $10.0 \mu \mathrm{L}$ was analyzed in triplicate. The peak areas of each compound in the sample solution were compared to the calibration curves, and the amount of each compound in $10 \mu \mathrm{L}$ of sample solution was calculated. The amount of compound 1 in $10.0 \mu \mathrm{L}$ of the sample solution (10.0 $\mu \mathrm{g}$ of fraction A) was calculated to be $0.332 \mu \mathrm{g}$, and its content in fraction $\mathrm{A}$ was $3.32 \%$. The amounts and contents of the other compounds were similarly calculated.

Preparation of primary cultured rat hepatocytes: Specific pathogen-free male Wistar rats were obtained from Charles River Laboratories Japan Inc. (Yokohama, Japan), housed at $21-23{ }^{\circ} \mathrm{C}$ on a 12 -h light-dark cycle, and fed a CRF-1 diet (Charles River Laboratories Japan) with water available ad libitum. The animals were acclimated for at least a week. Hepatocytes were isolated from the livers of the Wistar rats by collagenase perfusion [7]. The isolated cells resuspended in Williams' E medium were centrifuged and seeded at $1.2 \times 10^{6}$ cells per $35-\mathrm{mm}$ diameter dish. The hepatocytes were incubated at $37{ }^{\circ} \mathrm{C}$ for $2 \mathrm{~h}$. After the medium was changed, the hepatocytes were further incubated at $37{ }^{\circ} \mathrm{C}$ overnight for assays the following day. All animal care and experimental procedures were performed in accordance with the laws and guidelines of the Japanese government and were approved by the Animal Care Committee of Ritsumeikan University, Biwako-Kusatsu Campus (No. BKC2020-045).

\section{Measurement of NO levels and lactate dehydrogenase}

(LDH) activity: Each fraction or constituent was added to the hepatocyte medium with $1 \mathrm{nM}$ rat $\mathrm{IL}-1 \beta$, and the dishes were incubated at $37^{\circ} \mathrm{C}$ for $8 \mathrm{~h}$. The nitrite level (a stable metabolite of NO) in the medium was measured using the Griess method [8-9]. LDH activity in the medium was estimated using an LDH Cytotoxicity Detection kit (Takara Bio Inc., Otsu, Shiga, Japan) to monitor cytotoxicity. The half-maximal inhibitory concentration $\left(I_{50}\right)$ value of each fraction or constituent was calculated to determine its ability to suppress NO production unless the fraction or constituent was cytotoxic [9].

Direct NO quenching activity: Each fraction or constituent (dissolved in dimethyl sulfoxide) was added to medium containing $25 \mu \mathrm{M} \mathrm{NaNO}$ and incubated at $37^{\circ} \mathrm{C}$ for $1.5 \mathrm{~h}$ as previously described [10]. The final concentration of each constituent was $50 \mu \mathrm{M}$ (phellopterin) and $120 \mu \mathrm{M}$ (oxypeucedanin methanolate). The medium was then mixed with Griess reagent [9] and incubated at $23{ }^{\circ} \mathrm{C}$ for 5 min. The absorbance at $540 \mathrm{~nm}$ was measured to determine the reduction of nitrite.

Western blot analysis: Hepatocytes were treated with IL$1 \beta$ and each fraction or constituent at $37^{\circ} \mathrm{C}$, and wholecell lysates were prepared [11-12]. The lysates were resolved by sodium dodecyl sulfate-polyacrylamide gel electrophoresis (SDS-PAGE) and blotted onto Sequi-Blot membranes (Bio-Rad, Hercules, CA, USA). Immunostaining was performed using primary antibodies that were raised against rat iNOS (Thermo Fisher Scientific, Waltham, MA, USA) and rat $\beta$-tubulin (Cell Signaling Technology Inc., Danvers, MA, USA) and then a horseradish peroxidaseconjugated anti-immunoglobulin Fc antibody (Cell Signaling Technology Inc.). Protein was visualized using enhanced chemiluminescence blotting detection reagents (GE Healthcare Biosciences Corp., Piscataway, NJ, USA) and detected using an Amersham Imager 600 (GE Healthcare). To measure intensity of the bands, densitometry was performed using ImageJ software (http://imagej.nih. gov/ij/), and the band intensity of the iNOS was normalized by that of $\beta$-tubulin (internal control). The intensity of the iNOS band in the presence of IL-1 $\beta$ alone was set at 1 . 


\section{Quantitative reverse transcription-polymerase chain}

reaction (RT-PCR): Hepatocytes were treated with IL-1 $\beta$ and/or each constituent at $37^{\circ} \mathrm{C}$ for $4 \mathrm{~h}$, and total RNA was prepared [13]. The cDNA was reverse-transcribed with an oligo(dT) primer, and then quantitative PCR was performed with the primers for iNOS, elongation factor $1 \alpha$ (EF) [13], and tumor necrosis factor $\alpha$ (TNF- $\alpha$ ) mRNAs [14]. Relative mRNA levels were estimated in triplicate by quantitative PCR with a Thermal Cycler Dice Real Time system (Takara Bio Inc.), and the obtained values were normalized to EF mRNA, which was used as the internal control [13-14].

Statistical analysis: The results in the figures and tables are representative of at least three independent experiments that yielded similar results. The values are presented as the means \pm standard deviation (SD). Differences were analyzed using Student's $t$-test followed by Bonferroni's correction. Statistical significance was set at $P<0.05$ and $P<0.01$.
RESULTS

Inhibition of NO production by the crude fractions from the $A$. root extract: The roots of $A$. dahurica were extracted with $\mathrm{MeOH}$ and successively fractionated based on hydrophobicity with EtOAc (fraction A), $n$-butanol (fraction B), and water (fraction C), as shown in Figure 2. Then, the effects of the $A$. dahurica root extract and its crude fractions on NO production in hepatocytes in the presence of IL-1 $\beta$ were examined.

Among the crude fractions, the addition of fraction $\mathrm{A}$ to the medium significantly reduced IL- $1 \beta$-induced NO production in a dose-dependent manner. In contrast, fraction $B$ was less effective, and fraction $C$ did not cause a significant change in NO production (Figure 3A). Medium including each fraction showed much lower LDH activity than that of the whole cell extract (data not shown), suggesting that all fractions were not cytotoxic at the concentrations tested. Furthermore, Western blot analysis indicated that fraction $A$, as well as fraction $B$, suppressed the iNOS protein expression that was induced by IL-1 $\beta$ in hepatocytes (Figure 3B).

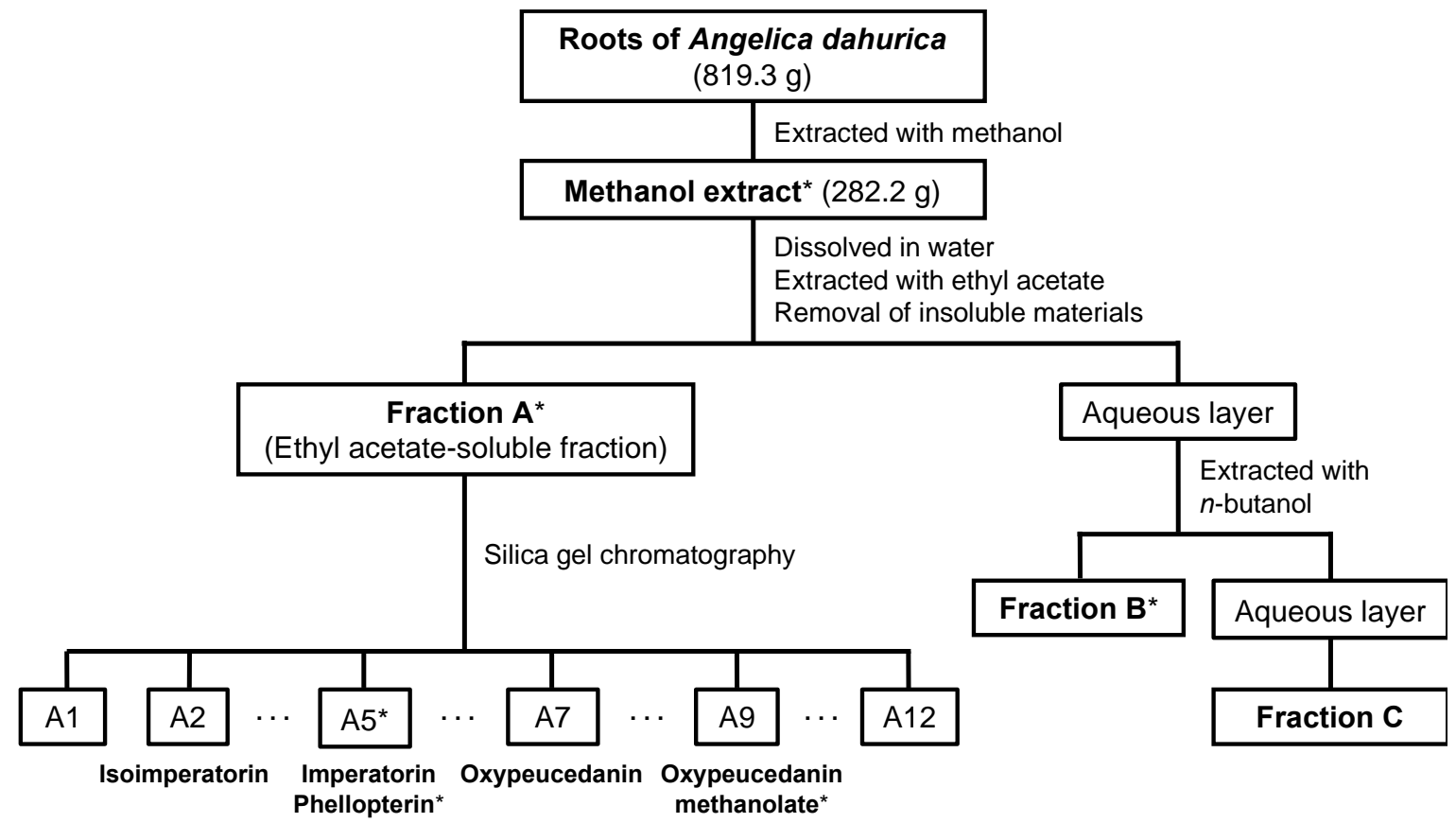

Figure 2. Isolation of the constituents from the root of $A$. dahurica. A flowchart of the procedure to isolate the constituents is shown schematically. The dried plant material was extracted and partitioned into three fractions based on hydrophobicity. The recovery of the extract from the dried roots was $34.4 \%$. Constituents isolated from fraction A are depicted under the relevant subfraction. * Extract, fraction, or constituent that suppressed NO induction in hepatocytes. 
A

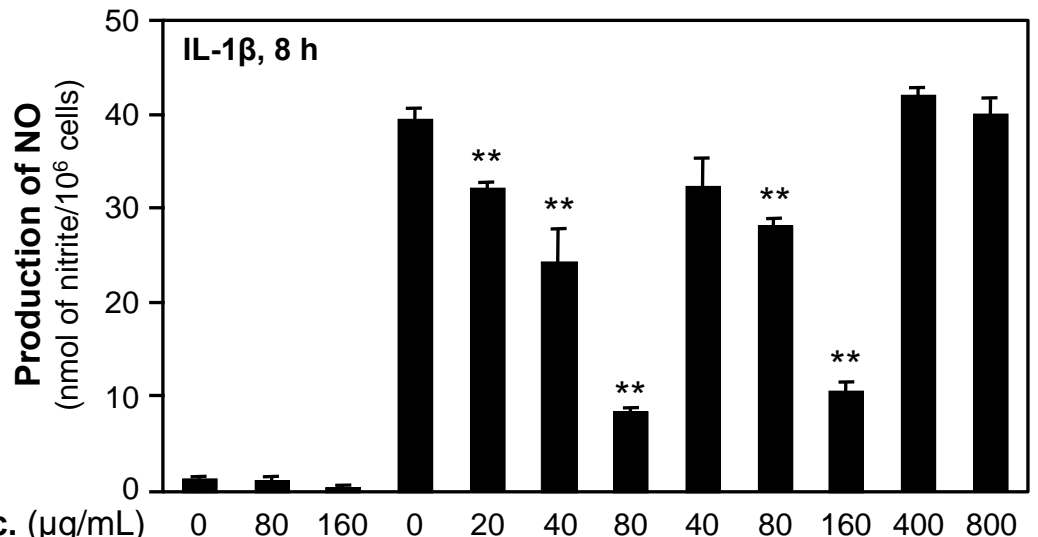

Conc. $(\mu \mathrm{g} / \mathrm{mL}) \quad 0 \quad 80 \quad 160 \quad 0 \quad 20 \quad 40 \quad 80 \quad 40 \quad 80 \quad 160 \quad 400 \quad 800$

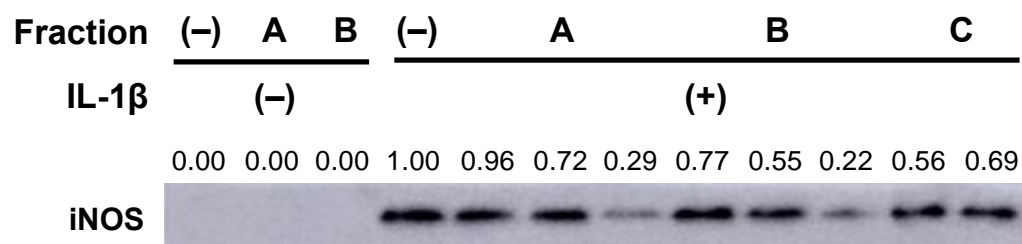

B

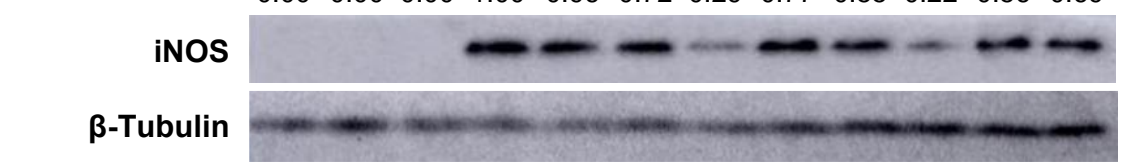

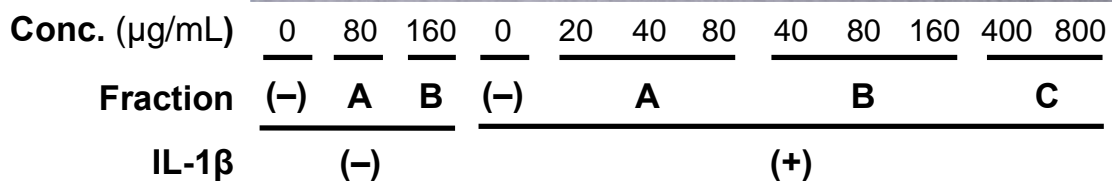

Figure 3. Fraction $A$ of the $A$. dahurica root extract suppressed NO and iNOS induction. (A) The effects of crude fractions $A$ to $C$ from an $A$. dahurica root extract on NO production. Hepatocytes were treated with $1 \mathrm{nM}$ IL-1 $\beta$ in the presence or absence of each fraction for 8 h. NO levels were measured by determining the amount of nitrite in the medium. The results represent the mean \pm SD $(n=3)$. When compared with the total LDH activity of the whole-cell extract (100\%), the LDH activity in each medium containing a fraction was very low (data not shown). ${ }^{*} P<0.05$ and $* * P<0.01$ versus IL-1 $\beta$ alone. (B) The effects of the fractions on iNOS expression. Hepatocytes were treated similarly to $(A)$. The cell extracts ( $20 \mu \mathrm{g}$ per lane) were subjected to Western blot analysis to detect iNOS ( $130 \mathrm{kDa})$ and $\beta$-tubulin ( $55 \mathrm{kDa}$; internal control). After densitometry, the band intensity of iNOS was normalized by that of $\beta$-tubulin and indicated by each lane.

Then, the $\mathrm{IC}_{50}$ values of the $A$. dahurica root extract and fractions $A$ and $B$ were calculated and are shown in Table 1. Fractions $A$ and $B$ showed lower $I_{50}$ values for NO production than the extract. Because fraction $A$ effectively suppressed NO production, it was expected that this fraction (the EtOAc-soluble fraction) may contain hydrophobic constituents that suppress IL-1 $\beta$-induced NO production.

\section{Isolation of the constituents of fraction $A$ from the $A$.} dahurica root extract: When fraction $A$ from the $A$. dahurica root extract was analyzed by HPLC, many peaks were observed (Figure 4A). Therefore, fraction A was fractionated into 12 subfractions by silica gel chromatography, which were further purified by silica gel chromatography and other methods, as described in the Materials and Methods. To determine the chemical structures of the purified constituents, we analyzed their ${ }^{1} \mathrm{H}$ - and ${ }^{13} \mathrm{C}-\mathrm{NMR}$ spectra (Supplementary Data) and compared them with the previously reported spectra of the relevant compounds [15-17]. Finally, we isolated five furanocoumarins and identified them as isoimperatorin (compound 1), imperatorin (3), phellopterin (4), oxypeucedanin (2), and oxypeucedanin methanolate (5) (Figure 4B). 
Table 1. Fractionation of an A. dahurica root extract and their effects on NO production.

$\begin{array}{lccc}\text { Fraction } & \text { Amount }[\mathrm{g}] & \text { Yield [\%] } & \text { IC }_{50}\left[\mathrm{Ig}^{\mathrm{a}} / \mathrm{mL}\right]^{\mathrm{b}} \\ \text { Methanol extract } & 282.2 & - & 254 \pm 38.4 \\ \text { A (ethyl acetate-soluble) fraction } & 27.6 & 10.7 & 25.7 \pm 4.0 \\ \text { B ( } n \text {-butanol-soluble) fraction } & 21.1 & 8.2 & 60.6 \pm 17.1 \\ \text { C (water-soluble) fraction } & 208.3 & 81.0 & \mathrm{NA}\end{array}$

${ }^{a}$ The percentage is calculated as the weight of each fraction divided by the sum of the three fractions (100\%). ${ }^{b}$ The half-maximal inhibitory concentration of NO production in IL-1 $\beta$-treated hepatocytes (mean \pm SD). At least three experiments were performed to determine these values. NA, not applied due to low activity.

A

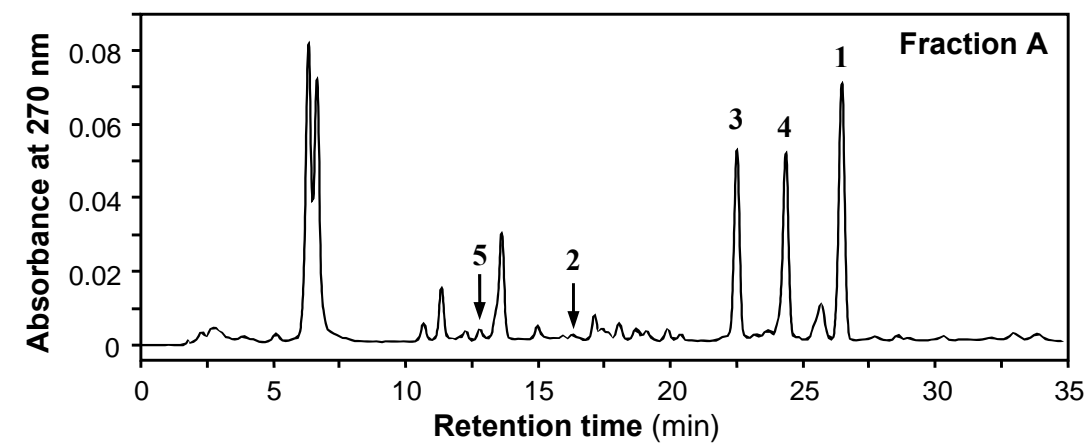

B

Isoimperatorin (1)
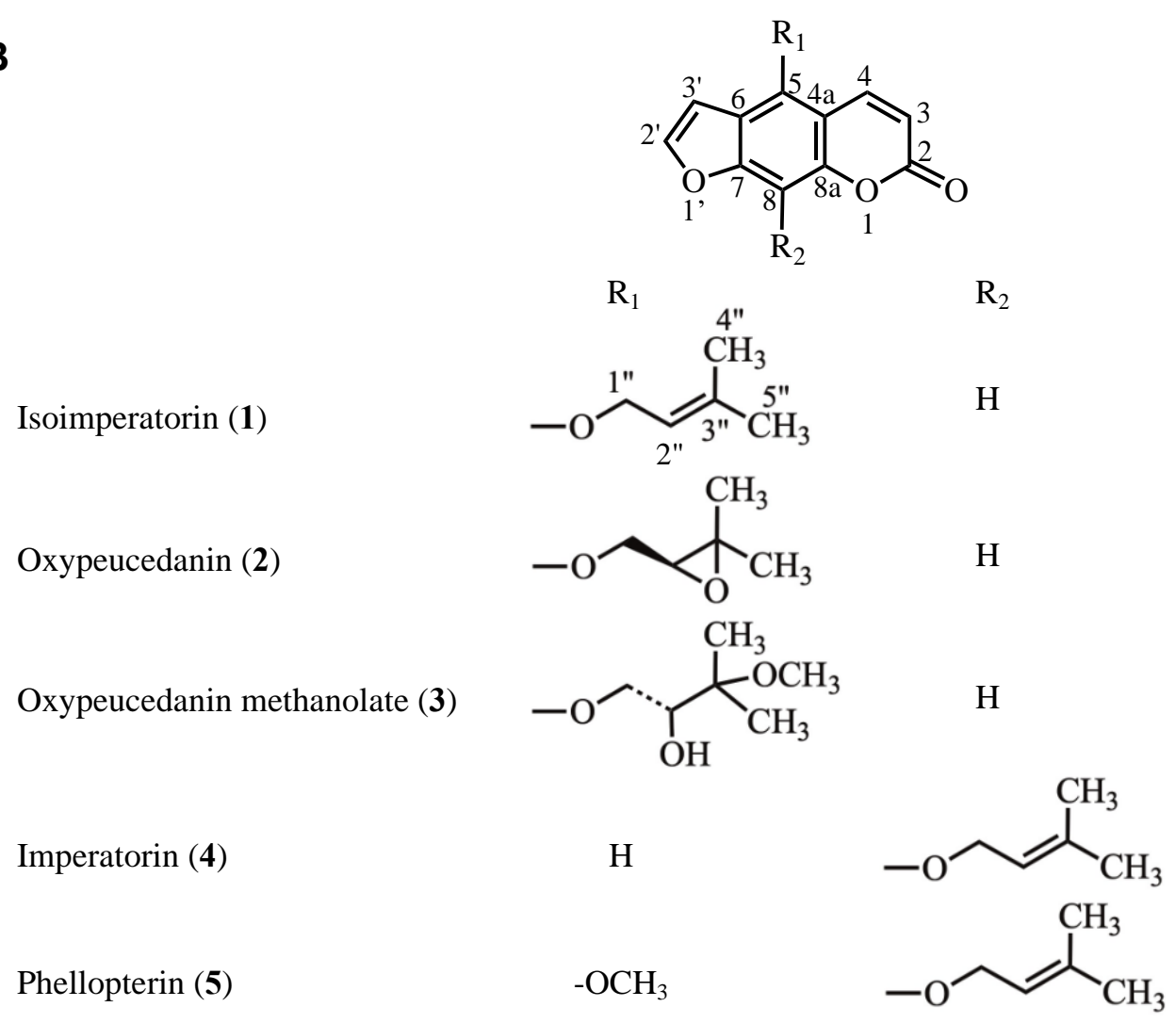

Figure 4. The furanocoumarins in fraction A from an A. dahurica root extract. (A) HPLC chromatogram of fraction A from the $A$. dahurica root extract. HPLC was performed using a Cosmosil 5C18 MS-II column. The UV detection wavelength was $270 \mathrm{~nm}$. The mobile phase was a binary eluent of water and acetonitrile, as described in the Materials and Methods. Each peak indicated by a number corresponds to the relevant compound number in (B). The retention times $\left(t_{R} \mathrm{~S}\right)$ of the peaks were $t_{R}=26.5 \mathrm{~min}$ (compound $\mathbf{1}$ ), $t_{\mathrm{R}}=16.3 \mathrm{~min}(\mathbf{2}), t_{\mathrm{R}}=22.5$ $\min (3), t_{R}=24.4 \min (4)$, and $t_{R}=12.8 \mathrm{~min}(5)$. (B) Chemical structures of the furanocoumarins in the $A$. dahurica root. 
Table 2. Constituents isolated from the A. dahurica root and their effects on NO production.

\begin{tabular}{llll}
\hline Constituent (Compound) & $\begin{array}{l}\text { Content } \\
{[\% \text { of fraction A }]^{\mathrm{a}}}\end{array}$ & $\begin{array}{l}\text { Content } \\
{[\% \text { of extract }]^{\mathrm{b}}}\end{array}$ & $\mathrm{IC}$ \\
Isoimperatorin (1) & 3.32 & 0.325 & $\mathrm{NA}$ \\
Imperatorin (3) & 3.23 & 0.316 & $\mathrm{NA}$ \\
Phellopterin (4) & 2.00 & 0.196 & $50.6 \pm 15.9$ \\
Oxypeucedanin methanolate (5) & 0.302 & 0.030 & $122 \pm 21.4$ \\
Oxypeucedanin (2) & 0.0988 & 0.010 & $\mathrm{NA}$
\end{tabular}

${ }^{a}$ The content of each constituent was measured by HPLC and is presented as a percentage of the dry weight of fraction A. ${ }^{b}$ The content of each constituent is shown as a percentage of the dry weight of the $\mathrm{MeOH}$ extract when $9.8 \%$ of fraction $\mathrm{A}$ was recovered from the $\mathrm{MeOH}$ extract. ${ }^{\mathrm{C}}$ The half-maximal inhibitory concentration of NO production in IL-1 $\beta$-treated hepatocytes (mean \pm SD). At least three experiments were performed to determine these values. NA, not applied due to low activity.

Next, HPLC analysis was performed to estimate the content of each constituent in fraction $A$. The contents of isoimperatorin (3.32\%) and imperatorin (3.23\%) were higher in fraction $A$ than the other constituents (Table 2).

\section{Inhibition of NO production by the constituents of}

fraction $A$ : We examined whether the furanocoumarins in fraction $A$ from an $A$. dahurica root extract could suppress NO induction in IL-1 $\beta$-treated hepatocytes. When isoimperatorin (1), imperatorin (3), and oxypeucedanin (2) were examined, these compounds did not affect NO production at final concentrations up to $340 \mu \mathrm{M}$ (data not shown). As shown in Figure 5A, phellopterin (4) and oxypeucedanin methanolate (5) significantly suppressed NO production in the presence of IL-1 $\beta$, whereas they did not induce NO production in the absence of IL-1 $\beta$. Neither furanocoumarin showed an increase in LDH activity in the medium (data not shown), which suggests that they are nontoxic. When the $\mathrm{IC}_{50}$ values were calculated, the value for phellopterin was the lowest, suggesting that phellopterin exhibited the highest potency to suppress NO among the five furanocoumarins tested (Table 2).

When the direct NO-quenching activities of phellopterin (4) and oxypeucedanin methanolate (5) were measured, no significant changes in NO levels were observed when compared with the NO levels in the medium containing $\mathrm{NaNO}_{2}$ alone (data not shown). These data imply that phellopterin and oxypeucedanin methanolate did not directly quench NO.

Inhibition of iNOS gene expression by phellopterin and oxypeucedanin methanolate: The effects of phellopterin (4) and oxypeucedanin methanolate (5) on iNOS gene expression were further investigated. When each furanocoumarin was added to the hepatocyte medium, the increase in iNOS protein expression that was induced by IL-1 $\beta$ was suppressed (Figure 5B).

Next, quantitative RT-PCR analysis of iNOS mRNA was performed using total RNA from hepatocytes, as described in the Materials and Methods. As shown in Figure 6A, the expression of iNOS mRNA increased in the presence of IL-1 $\beta$ alone (a positive control), whereas the iNOS mRNA level in the absence of IL-1 $\beta$ was much lower than that of the positive control (data not shown). When phellopterin or oxypeucedanin methanolate was added to the medium with IL-1 $\beta$, iNOS mRNA levels decreased in a dose-dependent manner. No amplification was observed when cells were individually treated with phellopterin or oxypeucedanin methanolate (data not shown). These results suggest that phellopterin and oxypeucedanin methanolate suppressed iNOS gene expression by reducing iNOS mRNA levels. 
A

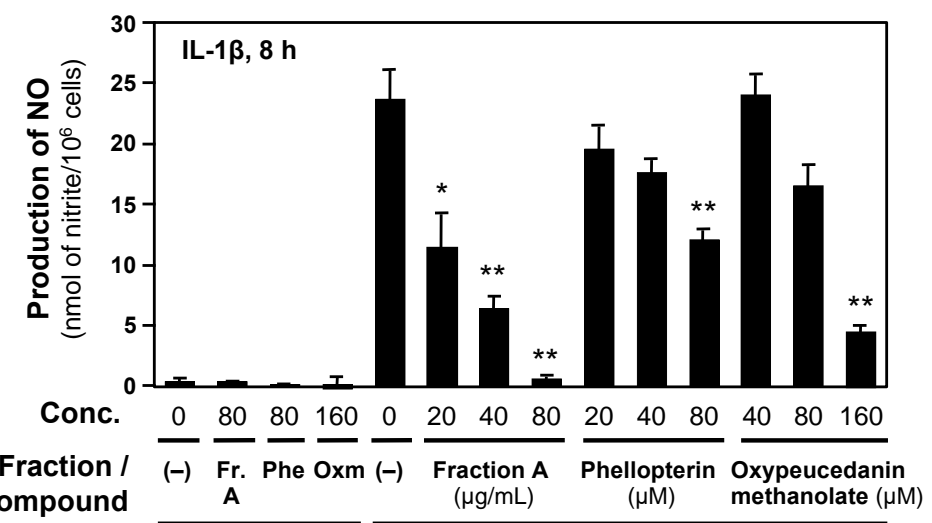

IL-1及

(-)

$(+)$

B

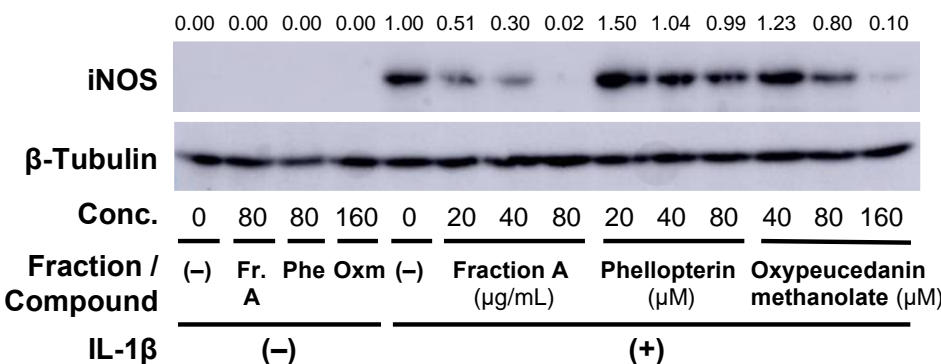

Figure 5. Phellopterin and oxypeucedanin methanolate suppress IL-1 $\beta$-induced iNOS gene expression. (A) The effects of phellopterin and oxypeucedanin methanolate on NO production. Hepatocytes were treated with $1 \mathrm{nM} \mathrm{IL-1 \beta}$ in the absence or presence of fraction A from the $A$. dahurica root extract, phellopterin (Phe), or oxypeucedanin methanolate $(\mathrm{Oxm})$ for $8 \mathrm{~h}$. Then, the NO levels in the medium were measured. The data represent the mean \pm SD $(n=3) . * P<0.05$ and $* * P<0.01$ versus IL- $1 \beta$ alone. (B) The effects of phellopterin and oxypeucedanin methanolate on iNOS protein levels. The hepatocytes were treated similarly to (A), and the cell extracts were subjected to Western blot analysis to detect the expression of iNOS and $\beta$-tubulin. After densitometry, the band intensity of iNOS was normalized by that of $\beta$-tubulin and indicated by each lane.

Furthermore, it was expected that phellopterin and oxypeucedanin methanolate might suppress other genes that are involved in inflammation. Therefore, we measured the expression levels of TNF- $\alpha$ mRNA in IL-1 $\beta$-treated hepatocytes. Quantitative RT-PCR analysis revealed that IL$1 \beta$ induced the TNF- $\alpha$ mRNA levels (Figure 6B). When

A

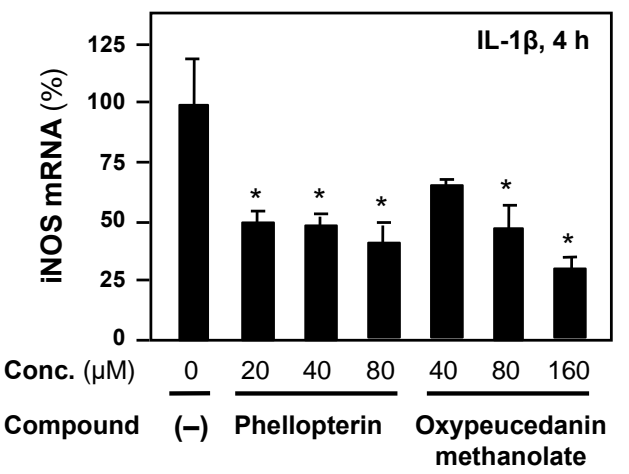

phellopterin or oxypeucedanin methanolate was added to the medium, both furanocoumarins decreased the expression of the TNF- $\alpha$ mRNA. No amplification was observed when cells were individually treated with phellopterin or oxypeucedanin methanolate (data not shown).
B

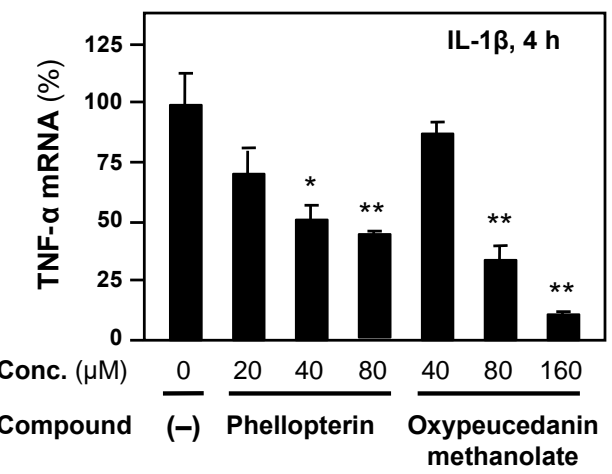

Figure 6. Phellopterin and oxypeucedanin methanolate inhibit the expression of proinflammatory genes. Total RNA was prepared following incubation with $1 \mathrm{nM} \mathrm{IL-1 \beta}$ in the presence of fraction A, phellopterin, or oxypeucedanin methanolate for $4 \mathrm{~h}$. Quantitative RTPCR was carried out to detect iNOS mRNA (A) and TNF- $\alpha$ mRNA (B) using EF mRNA as an internal control. The expression level of each mRNA was normalized to that of EF mRNA and is expressed as a percentage (mean $\pm \mathrm{SD}, n=3$ ). ${ }^{*} P<0.05$ and $* * P<0.01$ versus IL-1 $\beta$ alone. 


\section{DISCUSSION}

In the current study, five furanocoumarins were purified and identified from the hydrophobic fraction $(A)$ of an $A$. dahurica root extract. HPLC analyses provided the content of each constituent in the extract. The recovery rates were $34.4 \%$ of the $\mathrm{MeOH}$ extract from the dried roots (Figure 2) and $9.8 \%$ of fraction $A$ from the extract. Therefore, the content of imperatorin was calculated to be $0.109 \%$ in dried roots (Table 2). Previously, Pfeifer et al. reported that the dried roots of $A$. dahurica contained $0.09-0.28 \%$ imperatorin [18], which is comparable to our results. Similarly, the content of isoimperatorin $10.112 \%$ in the dried roots) is also comparable to the result from this report (0.04-0.10\%) [18].

Using primary cultured rat hepatocytes, we estimated the potencies of the constituents to suppress IL$1 \beta$-induced NO production to evaluate their antiinflammatory effects. Comparative analysis of the five constituents was performed using hepatocytes. The $\mathrm{IC}_{50}$ value of phellopterin (50.6 $\mu \mathrm{M})$ was comparable to values previously obtained (39.0 $\mu \mathrm{M}$ (luteolin from the leaves of Perilla frutescens Britton var. crispa form viridis) [19] and $51.0 \mu \mathrm{M}$ (nobiletin from the peel of Citrus unshiu [20])), suggesting that phellopterin may possess comparable potency to those compounds. The $I_{50}$ value of oxypeucedanin methanolate $(122 \mu \mathrm{M})$ was also comparable to the earlier determined values of $109 \mu \mathrm{M}(\gamma-$ schizandrin from the fruit of Schisandra chinensis) [11] and $129 \mu \mathrm{M}[(-)$-epicatechin) [21].

Phellopterin and oxypeucedanin methanolate efficiently suppressed NO production in hepatocytes, whereas isoimperatorin, imperatorin, and oxypeucedanin were less potent. Xanthotoxine and bergapten are present in the roots of Angelica acutiloba Kitagawa, the roots and rhizomes of Saposhnikovia divaricata (Turcz.) Schischk, and the roots and rhizomes of Glehnia littoralis Fr. Schmidt ex Miq. [22]. These two furanocoumarins, however, did not affect NO production in hepatocytes [22]. When their chemical structures were compared (Figure 4B), a methoxy group at carbon 5 and a side chain at carbon 8 , which are present in phellopterin, seem to be essential to suppress NO production. Although the content of phellopterin and oxypeucedanin methanolate was low, $A$. dahurica roots contain many other furanocoumarins, such as byakangelicin and byakangelicol, whose activity is not evaluated. It is likely that many furanocoumarins are involved in the anti-inflammatory activity of $A$. dahurica roots. More studies using the furanocoumarins are necessary to elucidate the structure-activity relationship of furanocoumarins in the future.

Macrophages produce various proinflammatory mediators, such as $\mathrm{NO}$ and $\mathrm{PGE}_{2}$. In LPS-treated rat peritoneal macrophages, imperatorin had the most potent inhibitory potency against $\mathrm{PGE}_{2}$ production among isoimperatorin, imperatorin, phellopterin, oxypeucedanin methanolate, and byakangelicin [23]. In IL-1 $\beta$-treated hepatocytes, phellopterin suppressed NO production, whereas imperatorin did not (Table 2). This result may be due to differences in cell types and signal transduction pathways. Indeed, the potency to suppress NO production by anti-inflammatory drugs is not the same as that of $P G E_{2}$ production in LPS-treated RAW264.7 cells [9].

As a pharmacokinetic study, we did not perform an experiment to orally administer phellopterin or oxypeucedanin methanolate to animals. In general, when a drug is orally administered, the drug concentration in blood rapidly becomes high and then gradually decreases. Because $A$. dahurica roots contain many potent furanocoumarins, a total concentration of these furanocoumarins in blood might be high enough to exhibit an activity to suppress NO production when an $A$. dahurica root extract is orally administrated to animals.

The present study suggests that phellopterin and oxypeucedanin methanolate reduced both the protein and mRNA levels of the iNOS gene in IL-1 $\beta$-treated hepatocytes, resulting in suppression of iNOS gene expression (Figures $5,6 A)$. These two furanocoumarins downregulated the mRNA expression of TNF- $\alpha$ (Figure 6B). Our previous reports suggest that transcription factors, i.e., nuclear factor $\mathrm{KB}(\mathrm{NF}-\mathrm{KB})$ and CCAAT/enhancer-binding protein $\beta$ 
$(C / E B P \beta)$, in the IL-1 signaling pathway are involved in the molecular mechanisms of the pharmacological activity of crude drugs in Kampo medicine [11,24]. Indeed, phellopterin and oxypeucedanin methanolate decreased the levels of type $1 \mathrm{IL}-1$ receptor mRNA in rat hepatocytes (data not shown). More studies are required to clarify the involvement of these transcription factors in this pathway.

There is a possibility that fraction $\mathrm{B}$, i.e., $n$-butanolsoluble fraction, also contains anti-inflammatory constituents. We selected fraction $A$, because its potency to suppress NO production was 2.36-fold higher than that of fraction B (Table 1). Fraction B contains amphipathic constituents, such as alkaloids and glycosides. Indeed, fraction $B$ of an $A$. dahurica root extract contained alkaloids (e.g., dahurine A), but not glycosides [25-26]. Such alkaloids may possess anti-inflammatory activity.

It is expected that several furanocoumarins from $A$. dahurica roots may exhibit various pharmacological activities other than anti-inflammatory effects. Han et al. reported that phellopterin, but not imperatorin or bergapten, promoted the differentiation of 3T3-L1 cells (a mouse fetal fibroblast cell line) to adipocyte-like cells [27]. Furthermore, phellopterin decreased blood glucose levels in type 2 diabetes mellitus model mice that were induced by high-fat diets and streptozotocin [27]. Recently, Tsai et al. reported that imperatorin inhibited cAMP-specific phosphodiesterase (PDE) 4 and alleviated psoriasiform dermatitis [28]. It is unknown whether phellopterin or oxypeucedanin methanolate has PDE activity. If they have PDE activity, they may contribute anti-inflammatory effects. Detailed studies of the furanocoumarins in $A$. dahurica roots and their pharmacological effects, including antidiabetic activity, remain to be investigated in the future.

\section{CONCLUSION}

The results of the current study on hydrophobic constituents suggest that two furanocoumarins, phellopterin and oxypeucedanin methanolate, may be responsible for the anti-inflammatory activities of $A$. dahurica roots by suppressing proinflammatory gene expression.

List of Abbreviations: NO: nitric oxide, IL: interleukin, $\mathrm{IC}_{50}$ : half-maximal inhibitory concentration, SD: standard deviation, i.d.: internal diameter, NMR: nuclear magnetic resonance, $t_{\mathrm{R}}$ : retention time.

Authors' Contributions: $\mathrm{YI}, \mathrm{MN}$, and $\mathrm{KT}$ designed the research; RO, HA, TO, YN, TI, and TS performed the experiments and analyzed the data; $\mathrm{MN}, \mathrm{YI}$, and SS prepared the manuscript; and all authors were involved in the performance of the experiments and preparation of the manuscript.

Competing Interests: HA performed this study as an undergraduate student of the College of Life Sciences, Ritsumeikan University. RO, TI, and TS performed the experiments as graduate students of the Graduate School of Life Sciences, Ritsumeikan University. YN performed the experiments as a graduate student of the Graduate School of Pharmaceutical Sciences, Ritsumeikan University. All authors have no financial interests or conflicts of interest.

Acknowledgments and Funding: The authors would like to thank Ms. Noriko Kanazawa for her secretarial assistance. This work was supported in part by the Asia-Japan Research Institute of Ritsumeikan Asia-Japan Research Organization, Ritsumeikan University.

\section{REFERENCES}

1. Ikeya Y, Epp DA, Nishizawa M: Anti-inflammatory effect of foods and crude drugs in relation to bitter and spicy tastes. Bioactive Compounds in Health and Disease 2019, 2: 77-93. https://doi.org/10.31989/bchd.v2i4.613

2. Colasanti M, Suzuki H: The dual personality of NO. Trends Pharmacol Sci 2000, 21: 249-252. https://doi.org/10.1016/S0165-6147(00)01499-1

3. Kitade H, Sakitani K, Inoue K, Masu Y, Kawada N, Hiramatsu Y, Kamiyama $\mathrm{Y}$, Okumura $\mathrm{T}$, Ito $\mathrm{S}$ : Interleukin $1 \beta$ markedly stimulates nitric oxide formation in the absence of other 
cytokines or lipopolysaccharide in primary cultured rat hepatocytes but not in Kupffer cells. Hepatology 1996, 23: 797-802.

https://doi.org/10.1053/jhep.1996.v23.pm0008666334

4. Nishizawa M, Okumura T, Ikeya $\mathrm{Y}$ : Assessment of antiinflammatory effects of Japanese Kampo medicine and functional foods. Functional Foods in Health and Disease 2019, 9: 79-91.

\section{https://doi.org/10.31989/ffhd.v9i2.566}

5. Kang OH, Lee GH, Choi HJ, Park PS, Chae HS, Jeong SI, Kim YC, Sohn DH, Park H, Lee JH, Kwon DY: Ethyl acetate extract from Angelica Dahuricae Radix inhibits lipopolysaccharide-induced production of nitric oxide, prostaglandin $E_{2}$ and tumor necrosis factor- $\alpha$ via mitogen-activated protein kinases and nuclear factor-KB in macrophages. Pharmacol Res 2007, 55: 263-270. https://doi.org/10.1016/j.phrs.2006.12.001

6. Ohno N, Yoshigai E, Okuyama T, Yamamoto Y, Okumura T, Sato $\mathrm{K}$, Ikeya Y, Nishizawa M: Chlorogenic acid from the Japanese herbal medicine Kinginka (Flos Lonicerae japonicae) suppresses the expression of inducible nitric oxide synthase in rat hepatocytes. HOAJ Biology 2012, 1: 2.

http://dx.doi.org/10.7243/2050-0874-1-2

7. Kanemaki $\mathrm{T}$, Kitade $\mathrm{H}$, Hiramatsu $\mathrm{Y}$, Kamiyama $\mathrm{Y}$, Okumura $\mathrm{T}$ : Stimulation of glycogen degradation by prostaglandin $E_{2}$ in primary cultured rat hepatocytes. Prostaglandins 1993, 45: 459-474. https://doi.org/10.1016/0090-6980(93)90122-N

8. Green LC, Wagner DA, Glogowski J, Skipper PL, Wishnok JS, Tannenbaum SR: Analysis of nitrate, nitrite, and $\left[{ }^{15} \mathrm{~N}\right]$ nitrate in biological fluids. Anal Biochem 1982, 126: 131-138. https://doi.org/10.1016/0003-2697(82)90118-X

9. Inaba H, Yoshigai E, Okuyama T, Murakoshi M, Sugiyama $\mathrm{K}$, Nishino $H$, Nishizawa M: Antipyretic analgesic drugs have different mechanisms for regulation of the expression of inducible nitric oxide synthase in hepatocytes and macrophages. Nitric Oxide 2015, 44: 61-70.

https://doi.org/10.1016/j.niox.2014.12.001

10. Tanemoto R, Okuyama T, Matsuo H, Okumura T, Ikeya $\mathrm{Y}$, Nishizawa M: The constituents of licorice (Glycyrrhiza uralensis) differentially suppress nitric oxide production in interleukin-1 $\beta$-treated hepatocytes. Biochem Biophys Rep 2015, 2: 153-159.

https://doi.org/10.1016/j.bbrep.2015.06.004

11. Takimoto $\mathrm{Y}$, Qian HY, Yoshigai E, Okumura T, Ikeya Y, Nishizawa M: Gomisin N in the herbal drug gomishi (Schisandra chinensis) suppresses inducible nitric oxide synthase gene via
C/EBP $\beta$ and NF-KB in rat hepatocytes. Nitric Oxide 2013, 28: 47-56. https://doi.org/10.1016/j.niox.2012.10.003

12. Nishizawa M, Kano M, Okuyama T, Okumura T, Ikeya Y: Antiinflammatory effects of enzyme-treated asparagus extract and its constituents in hepatocytes. Functional Foods in Health and Disease 2016, 6: 91-109.

https://doi.org/10.31989/ffhd.v6i2.228

13. Matsui K, Nishizawa M, Ozaki T, Kimura T, Hashimoto I, Yamada M, Kaibori M, Kamiyama Y, Ito S, Okumura T: Natural antisense transcript stabilizes inducible nitric oxide synthase messenger RNA in rat hepatocytes. Hepatology 2008, 47: 686697. https://doi.org/10.1002/hep.22036

14. Ningsih FN, Okuyama T, To S, Nishidono Y, Okumura T, Tanaka K, Ikeya Y, Nishizawa M: Comparative analysis of antiinflammatory activity of the constituents of the rhizome of Cnidium officinale using rat hepatocytes. Biol Pharm Bull 2020, 43: 1867-1875. https://doi.org/10.1248/bpb.b20-00416

15. Thanh PN, Jin WY, Song GY, Bae KH, Kang SS: Cytotoxic coumarins from the root of Angelica dahurica. Arch Pharm Res 2004, 27: 1211-1215.

https://doi.org/10.1007/BF02975883

16. Nizamutdinova IT, Jeong JJ, Xu GH, Lee SH, Kang SS, Kim YS, Chang KC, Kim HJ: Hesperidin, hesperidin methyl chalone and phellopterin from Poncirus trifoliata (Rutaceae) differentially regulate the expression of adhesion molecules in tumor necrosis factor- $\alpha$-stimulated human umbilical vein endothelial cells. Int Immunopharmacol 2008, 8: 670-678.

https://doi.org/10.1016/j.intimp.2008.01.011

17. Fujioka T, Furumi K, Fujii H, Okabe H, Mihashi K, Nakano Y, Matsunaga $H$, Katano $M$, Mori $M$ : Antiproliferative constituents from umbelliferae plants. V. A new furanocoumarin and falcarindiol furanocoumarin ethers from the root of Angelica japonica. Chem Pharm Bull (Tokyo) 1999, 47: 96-100. https://doi.org/10.1248/cpb.47.96

18. Pfeifer I, Murauer A, Ganzera M: Determination of coumarins in the roots of Angelica dahurica by supercritical fluid chromatography. J Pharm Biomed Anal 2016, 129: 246-251. https://doi.org/10.1016/j.jpba.2016.07.014

19. Nakajima A, Yamamoto Y, Yoshinaka N, Namba M, Matsuo H, Okuyama T, Yoshigai E, Okumura T, Nishizawa M, Ikeya Y: A new flavanone and other flavonoids from green perilla leaf extract inhibit nitric oxide production in interleukin $1 \beta$-treated hepatocytes. Biosci Biotechnol Biochem 2015, 79: 138-146. https://doi.org/10.1080/09168451.2014.962474

20. Yoshigai E, Machida T, Okuyama T, Mori M, Murase H, 
Yamanishi R, Okumura T, Ikeya $\mathrm{Y}$, Nishino $\mathrm{H}$, Nishizawa $\mathrm{M}$ : Citrus nobiletin suppresses inducible nitric oxide synthase gene expression in interleukin-1 $\beta$-treated hepatocytes. Biochem Biophys Res Commun 2013, 439: 54-59.

https://doi.org/10.1016/j.bbrc.2013.08.029

21. Yamanishi R, Yoshigai E, Okuyama T, Mori M, Murase $H$ Machida T, Okumura T, Nishizawa M: The anti-inflammatory effects of flavanol-rich lychee fruit extract in rat hepatocytes. PLoS One. 2014, 9: e93818.

https://doi.org/10.1371/journal.pone.0093818

22. Kamino T, Shimokura T, Morita $\mathrm{Y}$, Tezuka $\mathrm{Y}$, Nishizawa $\mathrm{M}$, Tanaka K: Comparative analysis of the constituents in Saposhnikoviae Radix and Glehniae Radix cum Rhizoma by monitoring inhibitory activity of nitric oxide production. J Nat Med 2016, 70: 253-259. https://doi.org/10.1007/s11418-0160969-1

23. Ban HS, Lim SS, Suzuki K, Jung SH, Lee $S$, Lee $Y S$, Shin $\mathrm{KH}$, Ohuchi K: Inhibitory effects of furanocoumarins isolated from the roots of Angelica dahurica on prostaglandin $\mathrm{E}_{2}$ production. Planta Med 2003, 69: 408-412. https://doi.org/10.1055/s2003-39702

24. Yamauchi Y, Okuyama T, Ishii T, Okumura T, Ikeya Y, Nishizawa M: Sakuranetin downregulates inducible nitric oxide synthase expression by affecting interleukin-1 receptor and CCAAT/enhancer-binding protein $\beta$. J Nat Med 2019, 73: 353368. https://doi.org/10.1007/s11418-018-1267-x

25. Qi B, Yang W, Ding N, Luo Y, Jia F, Liu X, Wang J, Wang X, Tu P, Shi S: Pyrrole 2-carbaldehyde derived alkaloids from the roots of Angelica dahurica. J Nat Med 2019, 73: 769-776. https://doi.org/10.1007/s11418-019-01328-1

26. Gao FY, Chen HY, Luo YS, Chen JK, Yan L, Zhu JB, Fan GR, Zhou TT: “Q-markers targeted screening” strategy for comprehensive qualitative and quantitative analysis in fingerprints of Angelica dahurica with chemometric methods. Food Chem X 2021, 12: 100162

https://doi.org/10.1016/j.fochx.2021.100162

27. Han HS, Jeon H, Kang SC: Phellopterin isolated from Angelica dahurica reduces blood glucose level in diabetic mice. Heliyon 2018, 4: e00577.

https://doi.org/10.1016/j.heliyon.2018.e00577

28. Tsai YF, Chen CY, Lin IW, Leu YL, Yang SC, Syu YT, Chen PJ, Hwang TL: Imperatorin alleviates psoriasiform dermatitis by blocking neutrophil respiratory burst, adhesion, and chemotaxis through selective phosphodiesterase 4 inhibition. Antioxid Redox Signal 2021, 35: 885-903.

https://doi.org/10.1089/ars.2019.7835 